\title{
Evidence for colliding winds in WR 25 from XMM-Newton observations of X-ray variability ${ }^{\star}$
}

\author{
A. M. T. Pollock ${ }^{1}$ and M. F. Corcoran ${ }^{2,3}$ \\ 1 European Space Agency, XMM-Newton Science Operations Centre, European Space Astronomy Centre, Apartado 50727, \\ Villafranca del Castillo, 28080 Madrid, Spain \\ 2 Laboratory for High Energy Astrophysics, Goddard Space Flight Center, Greenbelt, MD 20771, USA \\ 3 Universities Space Research Association, 7501 Forbes Blvd, Ste 206, Seabrook, MD 20706, USA
}

Received 23 May 2005 / Accepted 8 September 2005

\section{ABSTRACT}

The Wolf-Rayet star WR 25 in the Carina Nebula is a surprisingly bright X-ray source, and amongst the brightest WR stars in X-rays. It is a suspected binary star, though its binary nature has been a matter of controversy. We report here observations of WR 25 from the XMM-Newton archive which showed an increase in X-ray luminosity of more than a factor of two. The X-ray absorption also increased. Such a large change in X-ray output is unknown in single massive stars, though large changes in X-ray brightness are seen in binaries. The most likely explanation of the observed X-ray variability is as a result of colliding-wind emission in a moderately eccentric binary with a period of about 4 years. The next periastron passage is expected in 2007. The X-ray spectrum is similar to that of the archetype colliding-wind binary WR 140.

Key words. X-rays: stars - stars: Wolf-Rayet - stars: individual: WR 25 - stars: winds, outflows - stars: binaries: general

\section{Introduction}

WR 25, or HD 93162, is a rather controversial object in the Carina Nebula. It is one of 3 Wolf-Rayet stars in the Nebula, which is also home to the enigmatic object $\eta$ Carinae, and of the 3 it is by far the brightest in X-rays. The origin of the large $\mathrm{X}$-ray flux is uncertain, but suggestive of colliding-wind emission in a binary system (Pollock 1987; Raassen et al. 2003). However, the binary nature of the star has been a matter of debate. The star possesses an X-ray overluminosity of $L_{\mathrm{x}} / L_{\mathrm{bol}} \approx$ $20 \times 10^{-7}$ (Seward \& Chlebowski 1982) and a high X-ray temperature, similar to other known or suspected massive binaries, in contrast to values including $L_{\mathrm{x}} / L_{\mathrm{bol}} \approx 2 \times 10^{-7}$ typical of single massive stars. The optical spectrum is a composite of a diluted Wolf-Rayet emission-line spectrum of subtype WN6-7 and strong early-type absorption (Walborn et al. 1985). More recently, van der Hucht (2001) attributed a classification of WN6h+O4f to the star. However no radial velocity variations have ever been observed, indicating either a long-period or low-inclination binary, or a single star. The X-ray emission could arise from colliding-wind emission in a massive binary, or perhaps from the interaction of a strong wind from a single star with a nearby circumstellar cloud or dust lane, though

^ Based on observations obtained with XMM-Newton, an ESA science mission with instruments and contributions directly funded by ESA Member States and NASA.
Raassen et al. (2003) argued that the presence of Fe XXV emission in the X-ray spectrum was indicative of colliding-wind emission and hence binarity. On the other hand, as Raassen et al. (2003) noted, the X-ray luminosity of WR 25 had remained relatively stable over a 10 -year timespan, with variations $<15 \%$, and this lack of large variability in X-rays has been taken as evidence of the singular nature of the star.

Here we report a series of archived observations of WR 25 obtained with the European Photon Imaging Counter (EPIC) aboard XMM-Newton. In most of these observations the X-ray flux from WR25 in the EPIC band showed modest variations consistent with Raassen et al. (2003). However, more recently, there has been an episode of a few weeks during which both the X-ray luminosity and absorption of WR 25 temporarily increased considerably.

\section{Observations}

Table 1 lists the XMM-Newton observations considered here. The first five were reported by Raassen et al. (2003) while the remainder were obtained as part of a campaign to monitor the X-ray emission of $\eta$ Carinae, which is about $7^{\prime}$ from WR 25. The observations mostly follow a pattern in which a group of several was spread over a few days in seasons separated by about 6 months or a year until the $\eta$ Carinae periastron campaign, which covered a few weeks of the summer in 2003. Most 
Table 1. XMM-Newton EPIC observations of WR 25.

\begin{tabular}{|c|c|c|c|c|c|c|c|c|c|}
\hline Date & MJD & arget & rev & $\begin{array}{l}\text { MOS1 } \\
\mathrm{m}^{a}+\mathrm{f}^{b}\end{array}$ & $\begin{array}{c}\text { MOS1 } \\
\text { Count rate } \\
\left(\mathrm{s}^{-1}\right)\end{array}$ & $\begin{array}{l}\text { MOS2 } \\
\mathrm{m}^{a}+\mathrm{f}^{b}\end{array}$ & $\begin{array}{c}\text { MOS2 } \\
\text { Count rate } \\
\left(\mathrm{s}^{-1}\right)\end{array}$ & $\begin{array}{c}\mathrm{pn} \\
\mathrm{m}^{a}+\mathrm{f}^{b}\end{array}$ & $\begin{array}{c}\text { pn } \\
\text { Count rate } \\
\left(\mathrm{s}^{-1}\right)\end{array}$ \\
\hline $2000-07-26$ & 51751 & $\eta$ Carınae $^{n}$ & 0115 & $\mathrm{FF}+\mathrm{T}$ & $0.387 \pm 0.003$ & $\mathrm{SW}+\mathrm{T}$ & $0.439 \pm 0.004$ & $\mathrm{FF}+\mathrm{T}$ & $1.214 \pm 0.007$ \\
\hline $2000-07-27$ & 51752 & $\eta$ Carinae $^{k}$ & 0116_0112580701 & $\mathrm{FF}+\mathrm{T}$ & $0.384 \pm 0.006$ & $\mathrm{SW}+\mathrm{T}$ & $0.457 \pm 0.008$ & $\mathrm{FF}+\mathrm{T}$ & $1.237 \pm 0.014$ \\
\hline $2001-06-25$ & 52085 & WR $25^{k}$ & 0283_0112560101 & $\mathrm{FF}+\mathrm{T}$ & $0.463 \pm 0.006$ & $\mathrm{FF}+\mathrm{T}$ & $0.469 \pm 0.006$ & $\cdots$ & $\cdots$ \\
\hline 2001-06-28 & 52088 & WR $25^{k}$ & 0284_0112560201 & $\mathrm{FF}+\mathrm{T}$ & $0.460 \pm 0.004$ & $\mathrm{FF}+\mathrm{T}$ & $0.468 \pm 0.004$ & $15+1$ & $1.385 \pm 0.007$ \\
\hline $2001-06-30$ & 52090 & WR $25^{k}$ & 0285_01 & $\mathrm{FF}+\mathrm{T}$ & 0.400 & $\mathrm{FF}+\mathrm{T}$ & 0.4 & $\mathrm{FF}+\mathrm{T}$ & 1.38 \\
\hline $2003-01-25$ & 52664 & $\eta$ Carinae $^{c}$ & 0573_0145740101 & $\mathrm{FF}+\mathrm{T}$ & $0.368 \pm 0.008$ & $\mathrm{FF}+\mathrm{T}$ & $0.342 \pm 0.007$ & $\mathrm{SW}+\mathrm{T}$ & \\
\hline 2003-01-27 & 52666 & $\eta$ Carinae $^{c}$ & 0574_0145740201 & $\mathrm{FF}+\mathrm{T}$ & $0.365 \pm 0.008$ & $\mathrm{FF}+\mathrm{T}$ & $0.341 \pm 0.007$ & $\mathrm{SW}+\mathrm{T}$ & $\ldots$ \\
\hline 2003-01-27 & 52666 & $\eta$ Carinae $^{c}$ & 0574_0145740301 & $\mathrm{FF}+\mathrm{T}$ & $0.366 \pm 0.008$ & $\mathrm{FF}+\mathrm{T}$ & $0.353 \pm 0.007$ & $\mathrm{SW}+\mathrm{T}$ & \\
\hline 2003-01-29 & 52668 & $\eta$ Carinae $^{c}$ & 0575_0145740401 & $\mathrm{FF}+\mathrm{T}$ & $0.354 \pm 0.007$ & $\mathrm{FF}+\mathrm{T}$ & $0.346 \pm 0.007$ & $\mathrm{SW}+\mathrm{T}$ & \\
\hline 2003-01-29 & 52668 & $\eta$ Carinae $^{c}$ & 0575_0145740501 & $\mathrm{FF}+\mathrm{T}$ & $0.372 \pm 0.008$ & $\mathrm{FF}+\mathrm{T}$ & $0.333 \pm 0.007$ & $\mathrm{SW}+\mathrm{T}$ & $\ldots$ \\
\hline 2003-06-08 & 52798 & $\eta$ Carinae $^{c}$ & 0640_0160160101 & $\mathrm{SW}+\mathrm{T}$ & $0.487 \pm 0.004^{g}$ & $\mathrm{FF}+\mathrm{T}$ & $0.487 \pm 0.004^{g}$ & $\mathrm{SW}+\mathrm{T}$ & $\cdots$ \\
\hline 2003-06-19 & 52809 & $\eta$ Carinae $^{c}$ & $0643 \_0160160901$ & $\mathrm{SW}+\mathrm{T}$ & $0.093 \pm 0.002^{x}$ & $\mathrm{FF}+\mathrm{T}$ & $0.331 \pm 0.003^{x}$ & $\mathrm{SW}+\mathrm{T}$ & 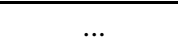 \\
\hline $2003-07-22$ & 52842 & $\eta$ Carinae $^{c}$ & 0662_0145780101 & $\mathrm{SW}+\mathrm{T}$ & $0.581 \pm 0.009$ & $\mathrm{FF}+\mathrm{M}$ & $0.672 \pm 0.009$ & $\mathrm{SW}+\mathrm{T}$ & $\cdots$ \\
\hline 2003-08-02 & 52853 & $\eta$ Carinae $^{c}$ & 0668_0160560101 & $\mathrm{SW}+\mathrm{T}$ & $0.346 \pm 0.005$ & $\mathrm{FF}+\mathrm{M}$ & $0.401 \pm 0.005$ & $\mathrm{SW}+\mathrm{T}$ & $\cdots$ \\
\hline 2003-08-09 & 52860 & $\eta$ Carinae $^{c}$ & 0671_0160560201 & $\mathrm{SW}+\mathrm{T}$ & $0.375 \pm 0.006$ & $\mathrm{FF}+\mathrm{M}$ & $0.442 \pm 0.006$ & $\mathrm{SW}+\mathrm{T}$ & $\ldots$ \\
\hline $2003-08-18$ & 52869 & $\eta$ Carinae $^{c}$ & 0676_0160560301 & $\mathrm{SW}+\mathrm{T}$ & $0.316 \pm 0.004$ & $\mathrm{FF}+\mathrm{M}$ & $0.433 \pm 0.005$ & $\mathrm{SW}+\mathrm{T}$ & $\ldots$ \\
\hline
\end{tabular}

${ }^{a}$ EPIC mode: FF full-frame mode; or SW small-window mode.

${ }^{b}$ EPIC filter: T Thick filter; or M Medium filter.

${ }^{c}$ PI: Corcoran.

${ }^{k}$ PI: Kaastra.

${ }^{g}$ Slightly affected by a MOS chip gap.

${ }^{x}$ Badly affected by a MOS chip gap.

of these observations were designed with $\eta$ Carinae in mind, with most of the EPIC-pn observations in small-window mode in which WR 25 was not visible. While nearly all the MOS1 and MOS2 observations provided high-quality data on WR 25, despite occasional bad columns and the proximity of chip gaps, in ObsIDs 0160160901 and 0160160901 the star fell squarely on chip gaps, in some exposures more seriously than others. With care these data are still useful, particularly for studying the spectrum given the energy independence of the PSF. Data were reduced using SASv6.0.0, using standard event selection criteria, and spectra extracted from a circle of radius 600 detector pixels centred on the star with the background estimated from a concentric annulus of inner and outer radii 1200 and 1800 pixels, respectively, using the SAS task especget, which also delivers the appropriate response and ancilliary response files.

\section{X-ray flux and absorption variations in WR 25}

It is obvious from the count rates alone reported in Table 1 that WR 25 showed considerable variability during the summer of 2003. Inspection of the raw spectra shows that this involved variations in both the intensity and the spectral shape, especially at low energies. By contrast, the spectrum did not change significantly over the few days covered by each observing season.
There are many possible ways to model the backgroundsubtracted MOS spectra. We found good fits to the data between 0.3 and $10.0 \mathrm{keV}$ with XSPEC (Arnaud 1996) using a single reference model subject to two extra degrees of freedom of luminosity and absorption. For the reference model, the most successful approach followed that of Raassen et al. (2003), who used a semi-empirical model consisting of two constant-temperature vmekal plasmas, each subject to independent wabs absorption. The best-fit reference model parameters, which are close to those reported by Raassen et al. (2003), are shown in Table 2 and describe the spectrum of WR 25 at its minimum luminosity and optical depth, defined most closely by Raassen et al. (2003)'s high statistical weight spectra taken over 5 days in June 2001. A large fraction of the observed absorption is due to the interstellar medium. Although Crowther et al. (1995) found that the extinction towards WR 25 is anomalous, their value of $E_{B-V}=0.66 \pm 0.03$ implies an interstellar column density of roughly $N_{\mathrm{H}} \sim 4 \times 10^{21} \mathrm{~cm}^{-2}$ which would account for most of the soft X-ray absorption in the reference spectrum. The apparently high nitrogen abundance, which is required by the model to account for a few counts at very low energy, is subject to large error and makes no difference to the results presented below.

Figure 1 shows a comparison of the MOS reference spectrum with the two spectra in which the luminosity and absorption reached their highest respective values. The systematic 




Fig. 1. Sum of XMM-Newton background-subtracted EPIC-MOS1 and EPIC-MOS2 spectra of WR 25 at 3 epochs: the low-luminosity, lowabsorption reference spectrum of 2001 June in red; the maximum-luminosity spectrum on 2003-07-22 in blue; the maximum-absorption spectrum on 2003-08-02 in green.

Table 2. Best-fit XSPEC reference model and abundances ${ }^{a}$ of $X M M$ Newton observations of WR 25

\begin{tabular}{|c|c|c|c|c|}
\hline \multicolumn{5}{|c|}{$\overline{A\left(\operatorname{wabs}\left(n \mathrm{H}_{1}\right) \operatorname{vmekal}\left(k T_{1}\right)+C_{12} \operatorname{wabs}\left(n \mathrm{H}_{2}\right) \operatorname{vmekal}\left(k T_{2}\right)\right)}$} \\
\hline$A$ & $=$ & 3.445 & \pm & $0.001 \times 10^{-3}$ \\
\hline$k T_{1}$ & $=$ & 0.609 & \pm & $0.005 \mathrm{keV}$ \\
\hline$n \mathrm{H}_{1}$ & $=$ & 7.17 & \pm & $0.12 \times 10^{21} \mathrm{~cm}^{-2}$ \\
\hline$C_{12}$ & $=$ & 0.523 & \pm & 0.036 \\
\hline$k T_{2}$ & $=$ & 2.774 & \pm & $0.041 \mathrm{keV}$ \\
\hline$n \mathrm{H}_{2}$ & $=$ & 5.07 & \pm & $0.35 \times 10^{21} \mathrm{~cm}^{-2}$ \\
\hline $\mathrm{He}$ & $=$ & $2.27^{b}$ & & \\
\hline $\mathrm{C}$ & $=$ & $0.15^{b}$ & & \\
\hline $\mathrm{N}$ & $=$ & 76. & \pm & 17. \\
\hline $\mathrm{O}$ & $=$ & 0.00 & \pm & 0.06 \\
\hline $\mathrm{Ne}$ & $=$ & 1.58 & \pm & 0.21 \\
\hline $\mathrm{Na}$ & $=$ & 7.5 & \pm & 1.9 \\
\hline $\mathrm{Mg}$ & $=$ & 1.41 & \pm & 0.17 \\
\hline $\mathrm{Al}$ & $=$ & 0.0 & \pm & 0.7 \\
\hline $\mathrm{Si}$ & $=$ & 1.46 & \pm & 0.16 \\
\hline$S$ & $=$ & 2.00 & \pm & 0.21 \\
\hline $\mathrm{Ar}$ & $=$ & 2.03 & \pm & 0.33 \\
\hline $\mathrm{Ca}$ & $=$ & 2.36 & \pm & 0.42 \\
\hline $\mathrm{Fe}$ & $=$ & 0.81 & \pm & 0.09 \\
\hline $\mathrm{Ni}$ & $=$ & 1.12 & \pm & 0.44 \\
\hline
\end{tabular}

${ }^{a}$ Relative to the default XSPEC solar abundances.

${ }^{b}$ Fixed value.

effects of changes are clear in luminosity at high energies and absorption at low energies. Despite this obvious overall variability, we found little evidence of any changes in the temperatures or luminosity ratio of the two components in any of the XMM-Newton observations, and so kept them and the minimum absorption fixed at the best-fit values to calculate the estimates of the history of WR 25's luminosity and additional absorption shown in Table 3 and plotted in Fig. 2 with details of the behaviour in 2003 shown in Fig. 3. MOS1 and MOS2 agree well although there is a tendency for MOS1 to yield values of $N_{\mathrm{H}}$ slightly larger by roughly $4 \times 10^{20} \mathrm{~cm}^{-2}$, due probably to remaining systematic uncertainties in the MOS calibration.

\section{Origin of the X-ray variability of WR 25}

The X-ray variability of WR 25 in the MOS spectra is similar to variations seen in other long-period, eccentric high-mass colliding-wind binaries in the following respects:

- a strong increase and subsequent decline in X-ray flux during a relatively brief interval;

- an accompanying increase in column density.

Given the optical spectrum and other circumstantial evidence about the duplicity of WR 25, the discovery of X-ray variability from the star almost conclusively identifies the star as a colliding-wind binary. The lack of radial velocity variations implies that WR 25 has a long period of some years. In eccentric systems of this type, X-ray variations can be produced by both changes in emission measure, expected to scale inversely with the binary separation, and changes in the amount of absorbing material along different lines-of-sight through the winds of the companion stars. Other long-period colliding-wind binaries, like WR 140, or suspected long-period CWBs, like $\eta$ Carinae, show a gradual increase in X-ray flux to a maximum followed 
Table 3. XMM-Newton EPIC-MOS X-ray history of WR 25.

\begin{tabular}{|c|c|c|c|c|c|c|}
\hline Date & MJD & rev_ObsID & $\begin{array}{c}\text { MOS1 } \\
F_{\mathrm{X}}^{a}\end{array}$ & $\begin{array}{c}\text { MOS1 } \\
N_{\mathrm{H}} \\
\left(10^{21} \mathrm{~cm}^{-2}\right)\end{array}$ & $\begin{array}{c}\text { MOS2 } \\
F_{\mathrm{X}}^{a}\end{array}$ & $\begin{array}{c}\text { MOS2 } \\
N_{\mathrm{H}} \\
\left(10^{21} \mathrm{~cm}^{-2}\right)\end{array}$ \\
\hline $2000-07-26$ & 51751 & 0115_0112580601 & $1.285 \pm 0.018$ & $0.41 \pm 0.08$ & $1.203 \pm 0.017$ & $0.03 \pm 0.08$ \\
\hline $2000-07-27$ & 51752 & 0116_0112580701 & $\begin{array}{l}1.325 \pm 0.032 \\
1.294 \pm 0.016\end{array}$ & $\begin{array}{l}0.69 \pm 0.15 \\
0.48 \pm 0.07\end{array}$ & $\begin{array}{l}1.273 \pm 0.033 \\
1.218 \pm 0.015\end{array}$ & $\begin{array}{l}0.16 \pm 0.15 \\
0.06 \pm 0.07\end{array}$ \\
\hline $2001-06-25$ & 52085 & 0283_0112560101 & $1.017 \pm 0.020$ & $0.71 \pm 0.12$ & $0.974 \pm 0.019$ & $0.00 \pm 0.22$ \\
\hline 2001-06-28 & 52088 & 0284_0112560201 & $0.985 \pm 0.012$ & $0.53 \pm 0.07$ & $1.003 \pm 0.012$ & $0.20 \pm 0.07$ \\
\hline 2001-06-30 & 52090 & 0285_0112560301 & $\begin{array}{l}1.013 \pm 0.012 \\
1.000 \pm 0.008\end{array}$ & $\begin{array}{l}0.75 \pm 0.07 \\
0.64 \pm 0.05\end{array}$ & $\begin{array}{l}1.009 \pm 0.012 \\
1.000 \pm 0.008\end{array}$ & $\begin{array}{l}0.22 \pm 0.07 \\
0.17 \pm 0.05\end{array}$ \\
\hline $2003-01-25$ & 52664 & 0573_0145740101 & $1.509 \pm 0.047$ & $2.01 \pm 0.21$ & $1.442 \pm 0.046$ & $1.65 \pm 0.22$ \\
\hline 2003-01-27 & 52666 & 0574_0145740201 & $1.363 \pm 0.043$ & $1.37 \pm 0.20$ & $1.335 \pm 0.043$ & $1.10 \pm 0.21$ \\
\hline $2003-01-27$ & 52666 & 0574_0145740301 & $1.401 \pm 0.044$ & $1.59 \pm 0.20$ & $1.425 \pm 0.046$ & $1.35 \pm 0.21$ \\
\hline 2003-01-29 & 52668 & 0575_0145740401 & $1.273 \pm 0.037$ & $1.11 \pm 0.18$ & $1.414 \pm 0.041$ & $1.48 \pm 0.19$ \\
\hline 2003-01-29 & 52668 & 0575_0145740501 & $\begin{array}{l}1.330 \pm 0.041 \\
1.368 \pm 0.019\end{array}$ & $\begin{array}{l}1.09 \pm 0.19 \\
1.41 \pm 0.09\end{array}$ & $\begin{array}{l}1.305 \pm 0.043 \\
1.384 \pm 0.020\end{array}$ & $\begin{array}{l}1.13 \pm 0.21 \\
1.34 \pm 0.09\end{array}$ \\
\hline 2003-06-08 & 52798 & 0640_0160160101 & $1.385 \pm 0.018^{g}$ & $0.20 \pm 0.08$ & $1.341 \pm 0.012^{g}$ & $0.00 \pm 0.04$ \\
\hline 2003-06-19 & 52809 & 0643_0160160901 & $0.914 \pm 0.027^{g}$ & $0.05 \pm 0.17$ & $1.026 \pm 0.010^{g}$ & $0.00 \pm 0.03$ \\
\hline $2003-07-22$ & 52842 & 0662_0145780101 & $2.912 \pm 0.063$ & $4.21 \pm 0.17$ & $2.453 \pm 0.049$ & $3.30 \pm 0.15$ \\
\hline 2003-08-02 & 52853 & 0668_0160560101 & $1.779 \pm 0.034$ & $4.82 \pm 0.16$ & $1.696 \pm 0.031$ & $4.56 \pm 0.15$ \\
\hline 2003-08-09 & 52860 & 0671_0160560201 & $1.673 \pm 0.037$ & $3.62 \pm 0.17$ & $1.682 \pm 0.035$ & $3.23 \pm 0.16$ \\
\hline $2003-08-18$ & 52869 & $0676 \_0160560301$ & $1.406 \pm 0.028$ & $2.02 \pm 0.14$ & $1.345 \pm 0.024$ & $1.56 \pm 0.11$ \\
\hline
\end{tabular}

${ }^{a}$ Ratio of the intersellar and circumstellar absorption-corrected 0.3-10. keV X-ray flux or luminosity to its 2001-06 minimum reference value.

${ }^{g}$ Affected by a MOS chip gap.
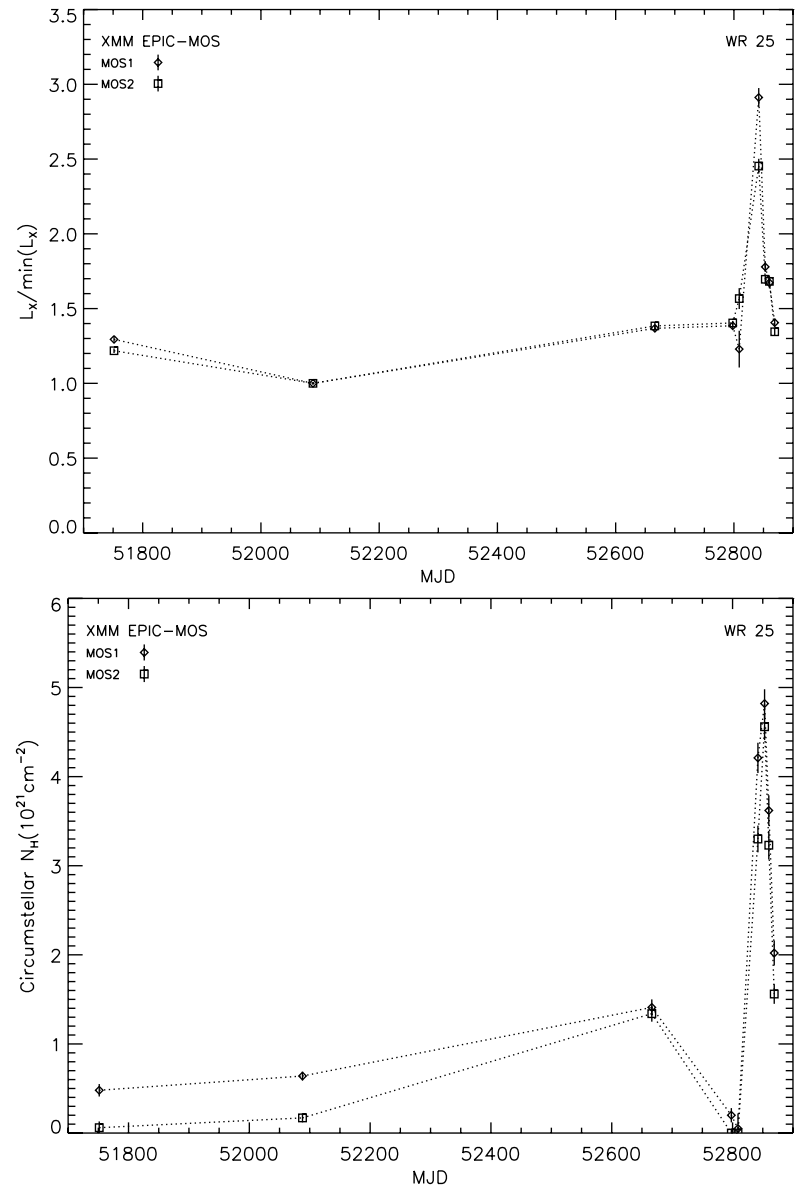

Fig. 2. The X-ray flux and absorption of WR 25 observed by XMMNewton between 2000-07-26 and 2003-08-18.
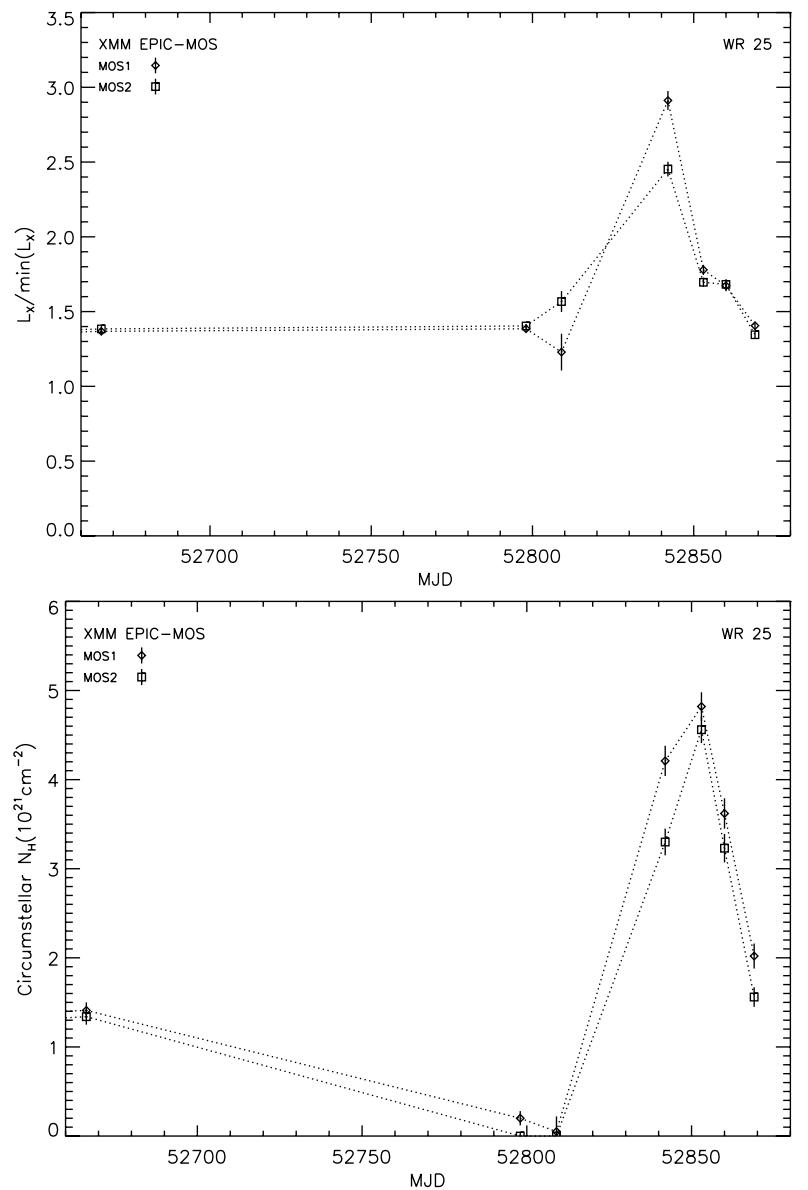

Fig. 3. Details of the X-ray flux and absorption of WR 25 observed by XMM-Newton during 2003. 
by a rapid decline associated, although not exactly coincident, with increasing column densities. Although the X-ray light curve of WR 25 has been sampled sparsely, several details support the proposal that the system is also an eccentric binary and give constraints on the orbital geometry:

- the maximum luminosity of 2003-07-22 marked the observation closest to periastron;

- the maximum luminosity ratio implies an eccentricity greater than about 0.5 ;

- the decrease of $20-30 \%$ in luminosity between 2000 July and 2001 June implies apastron occured in 2001, showing that the binary period is about 4 years;

- the maximum absorption observed 11 days after maximum luminosity marked superior conjunction of the more dense wind, presumably of the WR star;

- the low absorption of 2003 June a month or so before periastron marked quadrature;

- the intermediate absorption observed in 2003 January marked superior conjunction of the less dense wind, presumably of the $\mathrm{O}$ star;

The strongest X-ray variations of WR 25 have been confined to about 50 days of a supposed orbital period of perhaps 1500 days, giving a chance of only about 1 in 30 that a random observation falls sufficiently near periastron for variability to be detected. In retrospect, then, it is hardly surprising that none of previous observations made with a variety of X-ray instruments since WR 25's discovery in 1978 as a bright X-ray source have been successful in this respect. WR 25's X-ray spectrum shows obvious similarities to that of WR 140 described by Pollock et al. (2005), although it is a bit cooler. As pointed out by Raassen et al. (2003), lines are present in WR 25 from ionization stages of iron from Fe XVII at $15.015 \AA$ to Fe XXV at $1.850 \AA$, and there may even be a weak feature in Fig. 1 of
Fe XXVI in the spectrum nearest periastron. In WR 140 the variety of lines testifies to a plasma that is out of equilibrium (Pollock et al. 2005). The same is likely to apply to WR 25.

It is an interesting coincidence that a periastron passage of WR 25 seems to have occurred at the height of the campaign to cover the deep X-ray minimum of $\eta$ Carinae during its own periastron passage. This raises the possibility that some of the flux variability during $\eta$ Carinae's 2003 minimum detected by RXTE (Corcoran 2005) might have been due to WR 25 . However it is a possibility that, disappointingly, we have been able to exclude: folding the best-fit XMM models through the RXTE response shows that, even at its maximum, WR 25 was too weak to account for more than a few percent of the RXTE count rate.

On the basis of the arguments given above, the next periastron passage of WR 25 is expected in 2007, giving plenty of opportunity for the organization of a dedicated X-ray observing campaign.

\section{References}

Arnaud, K. A. 1996, Astronomical Data Analysis Software and Systems V, ed. G. Jacoby, \& J. Barnes, ASP Conf. Ser., 101, 17

Corcoran, M. F. 2005, AJ, 129, 2018

Crowther, P. A., Smith, L. J., Hillier, D. J., \& Schmutz, W. 1995, A\&A, 293, 427

van der Hucht, K. A. 2001, New Astron. Rev., 45, 135

Pollock, A. M. T. 1987, ApJ, 320, 283

Pollock, A. M. T., Corcoran, M. F., Stevens, I. R., \& Williams, P. M. 2005, ApJ, 629, 482

Raassen, A. J. J., van der Hucht, K. A., Mewe, R., et al. 2003, A\&A, 402, 653

Seward, F. D., \& Chlebowski, T. 1982, ApJ, 256, 530

Walborn, N. R., Nichols-Bohlin, J., \& Panek, R. J. 1985, NASA Reference Publication, 1155 